\title{
Integrated genomic analysis identifies the mitotic checkpoint kinase WEE1 as a novel therapeutic target in medulloblastoma
}

Peter S Harris ${ }^{1}$, Sujatha Venkataraman ${ }^{1}$, Irina Alimova', Diane K Birks², llango Balakrishnan ${ }^{1}$, Brian Cristiano ${ }^{1}$, Andrew M Donson ${ }^{1}$, Adrian M Dubuc ${ }^{4}$, Michael D Taylor ${ }^{4}$, Nicholas K Foreman ${ }^{1}$, Philip Reigan ${ }^{3}$ and Rajeev Vibhakar ${ }^{1 *}$

\begin{abstract}
Background: Medulloblastoma is the most common type of malignant brain tumor that afflicts children. Although recent advances in chemotherapy and radiation have improved outcomes, high-risk patients do poorly with significant morbidity.

Methods: To identify new molecular targets, we performed an integrated genomic analysis using structural and functional methods. Gene expression profiling in 16 medulloblastoma patient samples and subsequent gene set enrichment analysis indicated that cell cycle-related kinases were associated with disease development. In addition a kinome-wide small interfering RNA (siRNA) screen was performed to identify kinases that, when inhibited, could prevent cell proliferation. The two genome-scale analyses were combined to identify key vulnerabilities in medulloblastoma. The inhibition of one of the identified targets was further investigated using RNAi and a small molecule inhibitor.

Results: Combining the two analyses revealed that mitosis-related kinases were critical determinants of medulloblastoma cell proliferation. RNA interference (RNAi)-mediated knockdown of WEE1 kinase and other mitotic kinases was sufficient to reduce medulloblastoma cell proliferation. These data prompted us to examine the effects of inhibiting WEE1 by RNAi and by a small molecule inhibitor of WEE1, MK-1775, in medulloblastoma cell lines. MK-1775 inhibited the growth of medulloblastoma cell lines, induced apoptosis and increased DNA damage at nanomolar concentrations. Further, MK-1775 was synergistic with cisplatin in reducing medulloblastoma cell proliferation and resulted in an associated increase in cell death. In vivo MK-1775 suppressed medulloblastoma tumor growth as a single agent.
\end{abstract}

Conclusions: Taken together, these findings highlight mitotic kinases and, in particular, WEE1 as a rational therapeutic target for medulloblastoma.

Keywords: Medulloblastoma, WEE1, Mitosis, MK-1775, Integrated genomics

\footnotetext{
* Correspondence: rajeev.vibhakar@ucdenver.edu

'Department of Pediatrics and Section of Pediatric Hematology/Oncology/

BMT, Children's Hospital Colorado and University of Colorado Denver,

Anschutz Medical Campus, 12800 E 19th Ave, Mail Stop 8302, Aurora, CO

80045, USA

Full list of author information is available at the end of the article
} 


\section{Introduction}

Medulloblastoma is the most common malignant brain tumor in children. Over 800 cases per year occur worldwide with the vast majority occurring in children less than 12 years of age [1]. The mainstays of medulloblastoma therapy continue to be surgery, radiation and cytotoxic chemotherapy [2]. While therapy for standard risk patients has resulted in improved outcomes, high-risk patients do poorly. In particular those showing relapse and $M Y C$ amplification have a 5-year survival rate of less than $40 \%$ [3]. In addition, there remains significant therapy-related morbidity, particularly in the very young patients [4-6]. Novel therapeutic approaches based on tumor biology are clearly needed to improve outcomes for these children.

Recent genomic analysis has been successfully used to identify medulloblastoma subtypes [7-10]. International consensus has resulted in four molecular subgroups being defined [11]. These are the Wnt and Shh signaling subgroups as well as Group 3 and 4. Group 3 tumors largely represent the $M Y C$ amplified tumors whereas there is not a clear molecular definition of the Group 4 tumors [11]. However, finding therapeutic targets from these categories is still challenging [12]. Patients with the Wnt signaling signature are in a very good risk category and efforts are underway to de-escalate therapy for this cohort of patients [13]. For patients with the Shh signature, there are targeted inhibitors currently in early phase trials [13]. Unfortunately molecular targeting for Group 3 and 4 tumors is less clear. This is particularly problematic since Group 3 and 4 tumors constitute $60 \%$ of all medulloblastoma tumors [11].

The advent of RNA interference (RNAi) technologies for targeting large sets of genes in mammalian cells allows us to systematically interrogate gene functions in a high throughput manner $[14,15]$. This functional genomic approach has successfully resulted in the discovery of genes that were components of Ras oncogene driven tumors $[16,17]$, of genes that sensitize cells to chemotherapeutic agents [18], and of genes essential to the proliferation of such diverse cancer cells as neuroblastoma and renal cell carcinoma $[19,20]$.

Here we use an integrated descriptive and functional genomic analysis to identify molecular targets for medulloblastoma therapy. We performed pathway and gene set enrichment analysis on expression profiling data from 16 medulloblastoma samples to identify potential targetable pathways. In conjunction we performed a kinome-wide siRNA screen of medulloblastoma cells. Combined these results identified a set of mitotic-related kinases as potential therapeutic targets for medulloblastoma. We show that genetic and chemical inhibition of one of these kinases, WEE1, potently suppresses cell growth, induces apoptosis and decreases tumor volume in vivo in medulloblastoma. Further a small molecule inhibitor, MK-1775, acts in synergy with cisplatin to induce medulloblastoma cell death in vitro.

\section{Materials and methods}

\section{Cell lines and primary patient samples}

Dr. Darell D. Bigner (Duke University Medical Center, NC) kindly provided the D425 and D458 medulloblastoma suspension cell lines. The ONS-76 medulloblastoma cell line was graciously given by Dr. James $\mathrm{T}$. Rutka (University of Toronto, Canada) and the UW228 cell line by Dr. John Silber (University of Washington, Seattle). The Daoy and D283 medulloblastoma cell lines were purchased from American Type Cell Culture (Rockville, $\mathrm{MD}$ ). All cell lines were cultured in DMEM (Gibco, Carlsbad, CA) supplemented with 10\% fetal bovine serum (Atlanta Biologicals, Lawrenceville, GA).

The discovery cohort of 16 medulloblastoma patient samples used for gene expression profiling in Figure 1B and additional patient samples representing primitive neuroectodermal tumor, glioblastoma multiforme and pilocytic astrocytoma were obtained from Children's Hospital Colorado and was conducted in accordance with local and federal human research protection guidelines and Institutional Review Board (IRB) regulations. Informed consent was obtained for all specimens collected. Normal cerebellum collected from autopsy was purchased from Ambion (Austin, TX), Stratagene (Santa Clara, CA) and Clontech Laboratories, Inc. (Mountain View, CA). The other normal cerebellar samples (UPN 514 and UPN 605) were obtained from nonmalignant brain biopsies at the Children's Hospital Colorado under IRB guidelines. UPN 514 and UPN 605 are from 4 year old and 5 year old patients, respectively. The second large cohort of 90 tumor specimens were obtained in accordance with the Research Ethics Board at the Hospital for Sick Children (Toronto, Canada) and the N. N. Burdenko Neurosurgical Institute (Moscow, Russia) and data analysis was performed as described [7]. The second cohort was used to generate WEE1 gene expression array data for the normal cerebellum and the four distinct medulloblastoma molecular subgroups given in Figure 2C.

\section{Transfections with RNAi}

The siPORT NeoFX Transfection Agent purchased from Ambion was used to transfect the siRNAs targeting WEE1 mRNA (s21) and a non-targeting siRNA into medulloblastoma cell lines at a final concentration of $5 \mathrm{nM}$. The manufacturer's suggested protocol for a reverse transfection was used with the siRNA.

\section{Small molecule inhibitor of WEE1}

The small molecule WEE1 inhibitor, MK-1775, was purchased from Axon Medchem (Groninberg, Netherlands) 


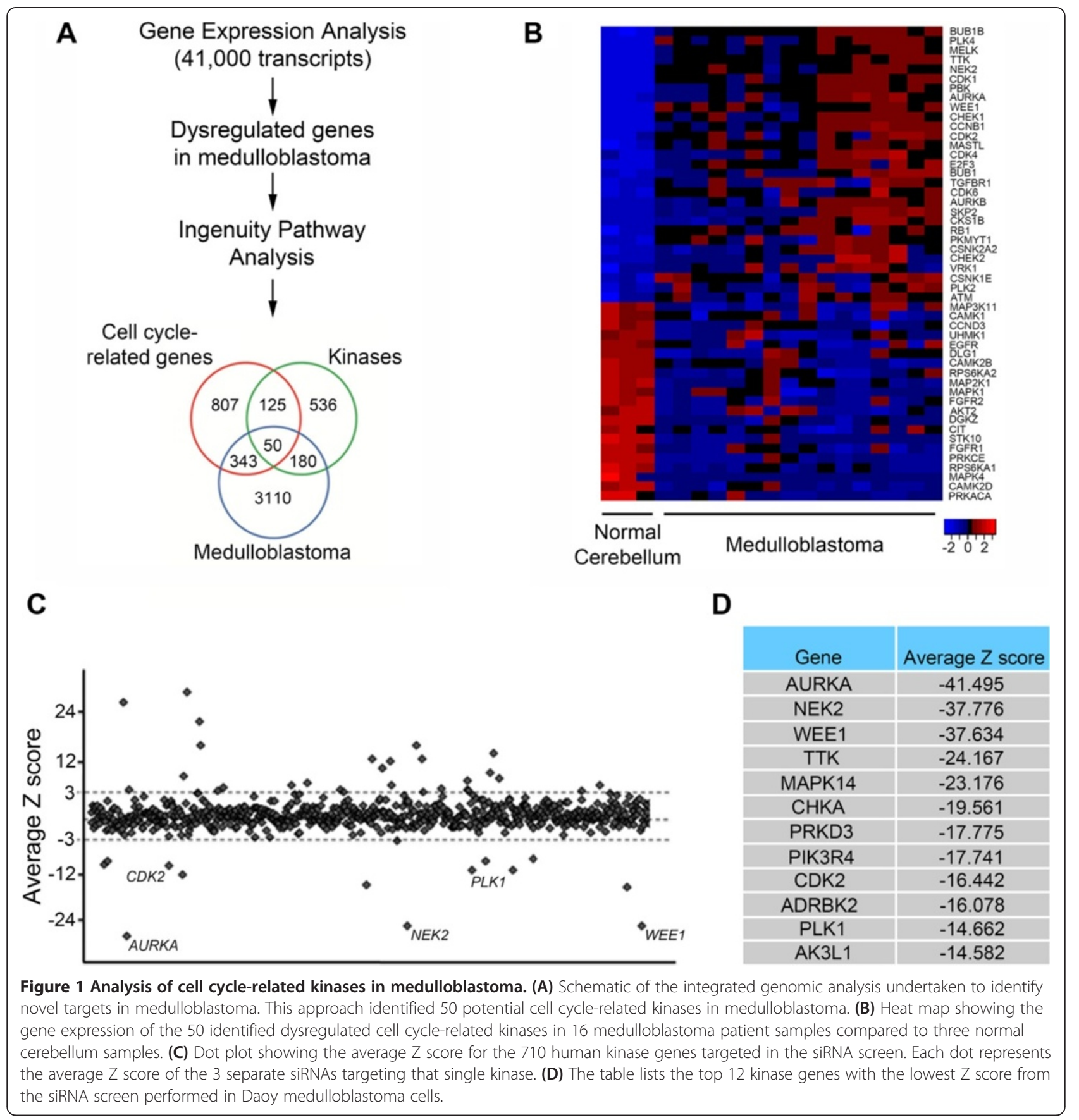

or synthesized by us. Dimethyl sulfoxide (DMSO) was used to reconstitute the drug and subsequent aliquots were stored in a desiccator at $-20^{\circ} \mathrm{C}$. An equivalent amount of DMSO for the highest concentration of drug was used for each experiment as a vehicle control.

\section{Cell proliferation assays}

Cell growth was measured using the xCELLigence system and E-Plate 96-well gold-coated plates (Roche, Indianapolis, IN). This system gives the real-time measurement of cell proliferation [21]. Cells were transfected with a siRNA against WEE1 or with a non-targeting siRNA control for 48 hours. Cells were then trypsinized and 2000 cells were plated in to a well of an E-plate and cell growth was measured.

Cell proliferation was determined by MTS [3-(4, 5dimethylthiazol-2-yl)-5-(3- carboxymethoxyphenyl)-2(4-sulfophenyl)-2H-tetrazolium] assay using CellTiter 96 AQueous One Solution (Promega, Madison, WI). For RNAi experiments $20 \mu \mathrm{L}$ of MTS reagent was added to 


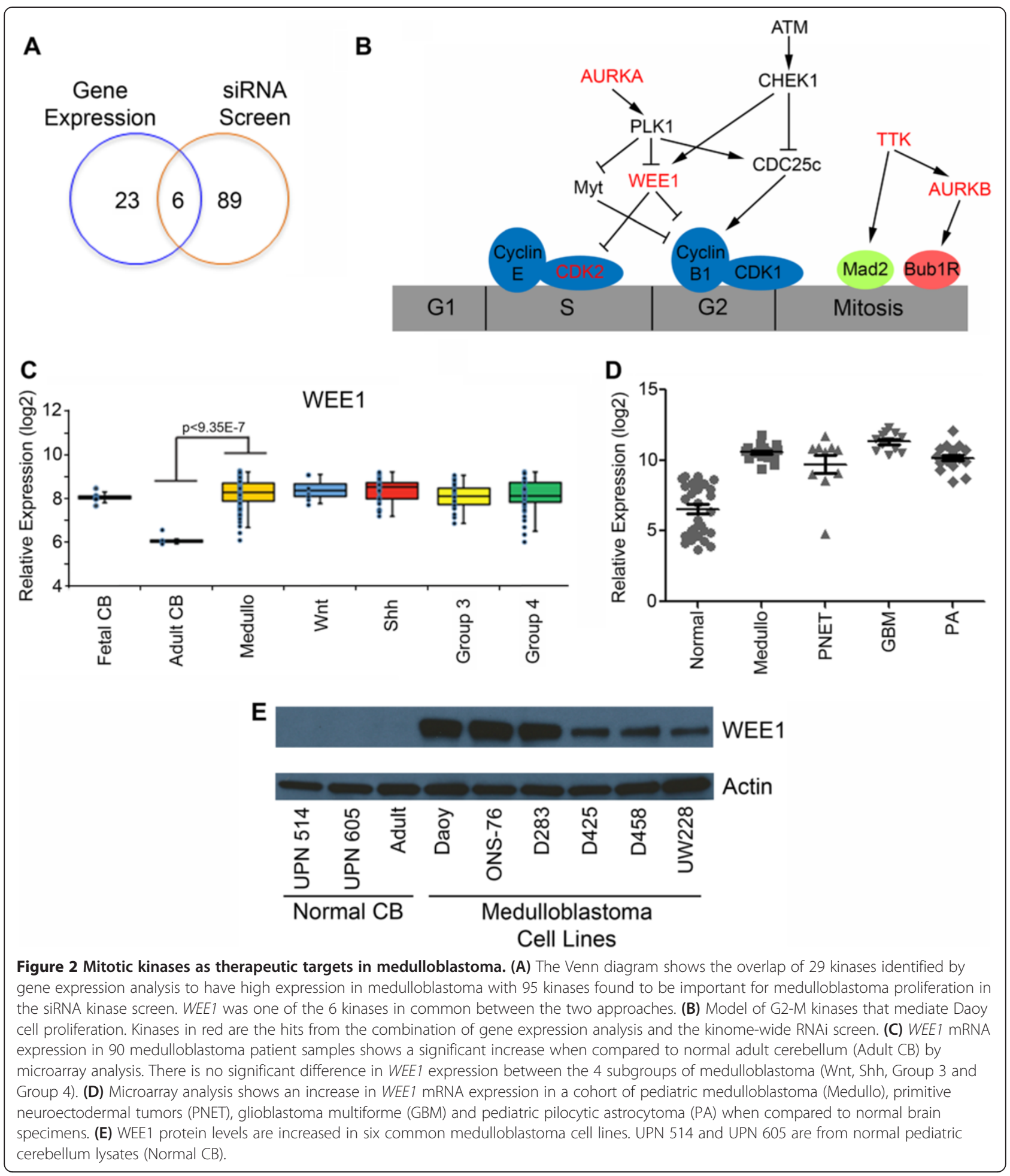

wells containing $100 \mu \mathrm{L}$ volumes seventy-two hours posttransfection. For chemical inhibitor experiments cells were plated for 24 hours before adding MK-1775. Then 72 hours after the addition of the drug, $30 \mu \mathrm{L}$ of MTS reagent was added to the wells to make a final volume of $180 \mu \mathrm{L}$. The optical densities of the triplicate wells were read using a BioTek Synergy 2 plate reader (Winooski, VT) every hour for 4 hours after the addition of the MTS reagent. Background absorbance was subtracted from all wells before analysis. The half maximal inhibitory concentration (IC50) values for the drug were calculated from the corrected absorbance values using GraphPad Prism. 


\section{Colony formation assay}

Cells were initially transfected with siRNA and then after 48 hours were counted and replated at 500 cells per well of a 6-well plate in triplicate. Colony formation assays utilizing MK-1775 were plated at the same density as above for 24 hours before drug addition. Wells were then treated with drug for 24 hours and subsequently allowed to grow in normal culture medium. Cells were allowed to grow for seven days, then the medium was aspirated, the wells were washed with phosphate buffered saline (PBS) and the colonies were stained with $0.5 \%$ crystal violet $/ 25 \%$ methanol solution. A dissecting microscope was used to count the number of colonies per well with a threshold of 50 cells necessary to constitute a colony.

\section{Cell apoptosis assay}

Cells were treated for 24 hours with drug and then allowed to grow in normal culture medium for an additional 24 hours. The cell concentration was determined following staining with Guava ViaCount reagent (Millipore, Billerica, MA). Equal numbers of cells were then stained using Guava Nexin reagent (Millipore) to detect apoptotic cells. Samples were run on a Guava EasyCyte Plus flow cytometer (Millipore).

\section{Immunofluorescence}

Three thousand cells grown in poly-D-lysine coated chamber slides were treated with either an IC25 of cisplatin, an IC30 of the WEE1 inhibitor MK-1775, cisplatin plus MK-1775 or DMSO for 6 hours or 24 hours. After treatment cells were washed and fixed with $4 \%$ paraformaldehyde for 15 minutes at room temperature. Cells were then permeabilized with $0.2 \%$ Triton X-100 in PBS for 15 minutes followed by incubation in 5\% milk diluted in $0.05 \%$ Triton X-100 for 30 minutes at room temperature on a shaker. After blocking, cells were incubated with the primary antibodies. The following antibodies were used at a dilution of 1:200: anti- $\gamma \mathrm{H} 2 \mathrm{AX}$ (Ser139), anti-phospho-H3 (Ser10) or anti-caspase 3 for 1 hour at room temperature. After washing with $0.05 \%$ Triton X-100 (3 times for 5 minutes each) cells were incubated with Alexa Fluor 488 conjugated secondary antibody (1:500) for 1 hour at room temperature in the dark, washed with PBS (3 times for 5 minutes each) and mounted using ProLong Gold antifade reagent containing DAPI (Invitrogen). Images were acquired using an inverted epifluorescence microscope at a magnification of 20x. At least three random fields were chosen to count cells containing greater than 10 foci.

\section{RNAi kinase screen}

A siRNA library containing 3 siRNAs per kinase for 710 human kinase genes was purchased from Ambion (catalog
\#4397918). The siRNAs in the 96-well plates were resuspended in nuclease-free sterile water to a concentration of $1 \mu \mathrm{M}$. Aliquots of the siRNAs were further diluted into daughter plates at a concentration of $250 \mathrm{nM}$. Daoy cells from the same passage were used to perform the screen. Briefly, Daoy cells were reverse transfected using siPORT NeoFX Transfection Agent with a final siRNA concentration of $5 \mathrm{nM}$. A separate triplicate set of wells containing cells transfected with a non-silencing siRNA were included on each plate. A MTS assay was performed 72 hours after transfection to determine whether the particular gene targeted by the siRNA had any effect on cell proliferation.

\section{Western blotting}

RIPA buffer (Thermo Scientific, Rockford, IL) containing protease inhibitors was used to obtain protein lysates. Western blotting was performed per standard methods. Primary antibodies purchased from Cell Signaling Technology that were used are WEE1 (\#4936), $\gamma$ H2AX (\#2577), phospho-CDK1 (Tyr15) (\#4539) and CDK1 (\#9112). The primary antibodies against actin (MAB1501) purchased from Millipore or $\beta$-tubulin (MAB1637) from Chemicon were used as loading controls. Secondary antibodies conjugated to horseradish-peroxidase were used in conjunction with a chemiluminescent reagent to visualize protein bands.

\section{Gene expression analysis}

Patient tumor samples were evaluated for gene expression using Affymetrix U133 Plus 2.0 Gene Chip microarrays as previously described [22]. Briefly, samples were collected at the time of surgery and snap-frozen in liquid nitrogen. RNA was extracted from each sample using an RNeasy kit (Qiagen, Valencia, CA, USA), reverse-transcribed and the resulting CDNA was converted to cRNA, and hybridized to HG-U133 Plus 2.0 GeneChips (Affymetrix) according to the manufacturer's instructions. Data analysis was performed in $\mathrm{R}$ (http://www.r-project.org/) using packages publicly available through Bioconductor (http://www.bioconductor.org). Hierarchical clustering was performed using the normalized gene expression data. Functional annotation analysis of differentially expressed genes was performed with the NIH Database for Annotation, Visualization, and Integrated Discovery (DAVID) Web tool (http://david.abcc.ncifcrf.gov/) using Biological Process Gene Ontology (GO) terms and Kyoto Encyclopedia of Genes and Genomes (KEGG) pathways. Gene set enrichment analysis was used to examine enrichment of genes in predefined reference sets that are based on biological knowledge [23]. Unlike other approaches that examine only genes meeting a predetermined cutoff, this tool computes an aggregate score for all genes in the reference set, based on their relative ranking in the data. Functional 
network analysis was performed using Ingenuity Pathways Analysis (IPA; Ingenuity Systems; http://www.ingenuity. com) which enables the visualization and exploration of molecular interaction networks on the basis of gene expression data. Networks were then algorithmically generated on the basis of their connectivity and were ranked by IPA on the basis of the number of genes represented in the network from the submitted gene list.

\section{In vivo xenograft analysis}

This study was conducted at Proxy Bioresearch, Inc. at its AAALAC accredited facility Proxy Bio, S.A. de C.V. The study was conducted following industry standards including compliance to United States Department of Agriculture and NIH animal care and use guidelines. Athymic nude mice were selected for this study because they have been used for similar tumor grafting studies and have been well-characterized. Daoy cell pellets were prepared using standard harvest procedures, resuspended in PBS and $7 \times 10^{7}$ cells were injected with $50 \%$ Matrigel by volume (BD Matrigel) using a $1 \mathrm{~mL}$ syringe and 18 gauge $1 / 2$ inch needle into the flank of each mouse in an approximately $200 \mu \mathrm{L}$ volume. Once tumors were palpable and measurable (mean 52 days post inoculation) treatment with MK-1775 or the control vehicle of DMSO was begun.

Control or MK-1775 (30 mg/kg) was dosed for 3 consecutive days every 7 days for 3 weeks by oral gavage and then followed for an additional 42 days with no therapy given. Each animal was tracked individually for tumor growth by external caliper measurements of subcutaneous protruding tumor and an approximate tumor volume was calculated using the ellipsoid volume formula: $\pi / 6 \times \mathrm{L} \times \mathrm{W} \times \mathrm{H}$. Animals were also weighed 3 times a week for the duration of the treatment. Upon termination of the study the tumors were excised and snap frozen in liquid nitrogen.

\section{Cell cycle analysis}

Cells were treated with an IC30 of MK-1775, an IC25 of cisplatin, or both for 24 hours. Cells were then harvested, washed with PBS and fixed in 70\% ethanol. Twenty-four hours after fixing cells were washed with PBS and then resuspended in Guava Cell Cycle reagent and analyzed on a Guava EasyCyte Plus flow cytometer.

\section{Statistics}

The $\mathrm{p}$ value for the gene expression of the second large cohort of 90 patient samples was calculated using the Mann-Whitney $U$ test. The remaining $p$ values were calculated using the Student's t test. The error bars represent the standard error of the mean.

\section{Results}

Cell cycle-related kinases are differentially expressed in medulloblastoma

As a first step to identify novel molecular targets we performed gene expression profiling on 16 medulloblastoma samples and on corresponding normal cerebellar tissue. The tumor samples were obtained at our institution at the time of surgery and represent all major molecular subgroups as previously described by us [24]. Gene expression was measured by Affymetrix microarrays [24]. We selected for dysregulated genes with a two-fold or greater difference between medulloblastoma and normal cerebellum (FDR 0.1). A total of 3683 transcripts were differentially expressed in medulloblastoma. To identify specific signaling networks we performed pathway analysis using IPA software (Ingenuity) and gene set enrichment analysis. Cell cycle-related genes were the most abundant in the molecular category and kinases were the most abundant in the functional category. By comparing the molecular and functional categories with the total dysregulated genes in medulloblastoma we found 50 specific genes that were common to all three categories (Figure 1A). The expression of these cell cyclerelated kinases is shown in the heat map in Figure 1B and in Additional file 1: Table S1. Twenty-nine of the fifty dysregulated cell cycle-related kinases are significantly over expressed compared to normal cerebellum.

\section{Role of protein and lipid kinases in medulloblastoma cell proliferation}

Based on our initial data that a subset of kinases are over expressed in medulloblastoma we carried out a kinomewide siRNA screen to identify kinases that are essential for medulloblastoma cell proliferation. The well characterized Daoy cell line was chosen as we have significant previous experience in manipulating it [24,25]. Daoy cells were transfected with 2130 unique siRNAs targeting each of 710 kinase genes or a non-silencing control in a 96 well format. Cell proliferation was evaluated by the MTS assay (CellTiter AQueous) after 72 hours of transfection. Absorbance values were normalized to reference controls on each plate to allow plate to plate comparison and the average $\mathrm{Z}$ score was calculated (Figure $1 C$ ). A $\mathrm{Z}$ score of $<-2$ was considered effective on decreasing medulloblastoma proliferation. A total of 95 genes were identified that when inhibited decreased Daoy cell growth (Figure $1 \mathrm{C}$ and Additional file 2: Table $\mathrm{S} 2$ ). Fifty genes met our criteria when we use a more stringent cutoff of $Z<-3$ with the 12 genes pertaining to the lowest $Z$ score shown in Figure 1D. Of these we have previously reported on polo-like kinase 1 and aurora kinase A $[25,26]$. We subsequently validated five of these genes in an independent siRNA screen for their effect on medulloblastoma cell proliferation (Additional file 3 : 
Figure S1). Of note as expected not all siRNA resulted in a biological phenotype due to the variability of siRNA knockdown in cells.

\section{Mitotic kinases are key determinants of medulloblastoma cell growth}

To integrate our gene expression data and our siRNA screen we compared the two sets of data. The 29 genes over expressed from the gene expression data was compared to the 95 kinases identified to inhibit cell proliferation by the siRNA screen. Only 6 genes are over expressed in medulloblastoma and also suppress medulloblastoma cell growth. These are aurora kinase A, WEE1, TTK, aurora kinase $B, C D K 2$ and PLK1 (Figure 2A). Interestingly all 6 genes are involved in the G2-M transition during the cell cycle (Figure 2B). Our previous report on the importance of aurora kinase A in medulloblastoma cell proliferation further validates this new methodology of identifying genes by integrated genomic analysis $[26,25]$. We chose to further examine the role of WEE1 kinase because it has recently been reported to be involved in several tumors including glioblastoma [27]. WEE1 regulates the G2-M cell cycle checkpoint by preventing DNA damaged cells from entering mitosis. We first examined the expression of WEE1 in an independent cohort of 90 medulloblastoma samples. Comparison of normal cerebellum with medulloblastoma showed significant over expression of WEE1 $(\mathrm{p}<9.35 \mathrm{E}-7)$ in this disease. However there was no significant difference in WEE1 expression between the recently identified subgroups as shown in Figure 2C. Next WEE1 expression was examined in a panel of pediatric brain tumors of differing pathological grade. WEE1 was over expressed in all three high-grade tumors examined including medulloblastoma (medullo), primitive neuroectodermal tumor (PNET) and glioblastoma multiforme (GBM). Interestingly WEE1 was also expressed in the low-grade pilocytic astrocytoma (PA) in which there is very little proliferation (Figure 2D). These data suggested that increased WEE1 expression may be a key to tumorigenesis and not simply a marker of proliferation. We next evaluated WEE1 protein in two normal pediatric cerebellum samples, one adult normal cerebellum sample and in a panel of well characterized medulloblastoma cell lines. WEE1 protein is not present in pediatric (UPN 514,605) or adult cerebellum but was present in varying amounts in the 6 medulloblastoma cell lines evaluated (Figure 2E).

\section{Inhibition of WEE1 suppresses medulloblastoma cell proliferation and colony forming potential in vitro}

Daoy and UW228 cell lines were chosen to examine the functional consequence of inhibiting WEE1. Using a siRNA against WEE1 we measured cell proliferation using the xCELLigence real-time cell analysis (RTCA) system. Cell growth was monitored following transfection of either a non-silencing siRNA or one targeting WEE1. A decrease in cell growth was seen in both the Daoy and UW228 cell lines (Figure 3A). The more pronounced decrease in growth seen in Daoy cells compared to UW228 cells could be explained by the higher expression of WEE1 protein in Daoy cells compared to the UW228 cell line (Figure 2E). We then chose to determine the ability of medulloblastoma cells to undergo an unlimited number of divisions following inhibition of WEE 1 by performing a colony forming assay. The siRNA targeting WEE1 showed a significant decrease in the relative colony number when compared to the non-silencing siRNA in both Daoy and UW228 cell lines (Figure 3B). Western blots confirmed the specificity and ability of the siRNA targeting WEE1 to decrease the WEE1 protein levels in Daoy and UW228 cells (Figure 3C).

Small molecule inhibitor of WEE1, MK-1775, potently suppresses medulloblastoma growth in vitro and in vivo Recently several inhibitors of WEE1 have been described [28-30]. We chose to examine MK-1775, a pyrazolopyrimidine derivative, which has recently entered Phase I/II trials in adult cancers. Daoy and UW228 cells were exposed to various concentrations of MK-1775. The IC50 values from the MTS assay were $150 \mathrm{nM}$ and $232 \mathrm{nM}$ for Daoy and UW228 cell lines respectively (Figure 4A). These nanomolar concentrations have been shown previously to be achievable in vivo and therefore make MK-1775 a clinically viable drug [31].

To evaluate the impact of MK-1775 on the long-term proliferative capacity in medulloblastoma cells, colony forming assays were performed in both Daoy and UW228 cell lines. In both cell lines 48 hour exposure to an IC30 of MK-1775 was sufficient to significantly decrease the colony number counted after 1 week ( $<<0.01$, Figure $4 \mathrm{~B})$. Furthermore, the Daoy cell line showed a significant decrease in relative colony number after exposure to an IC15 of MK-1775 ( $\mathrm{p}<0.01)$. UW228 cells did not show a further decrease in colony formation from the IC30 to IC15, which likely reflects the heterogeneity of tumor cells. Interestingly UW228 are p53 sufficient while Daoy cells are not. Clearly the role of p53 status on MK-1775 activity in medulloblastoma needs to be further examined. To assess whether MK-1775 was inhibiting WEE1 kinase activity, we measured the phosphorylation status of its target CDK1 by immunoblotting. A decrease in phosphoCDK1 (Tyr15) was seen in a dose-dependent manner with MK-1775 treatment as shown in Figure 4C. There was no significant change in WEE1 with MK-1775 treatment as expected.

We next examined the efficacy of MK-1775 in treating medulloblastoma tumors in vivo. Athymic nude mice were injected with Daoy cells subcutaneously and palpable 


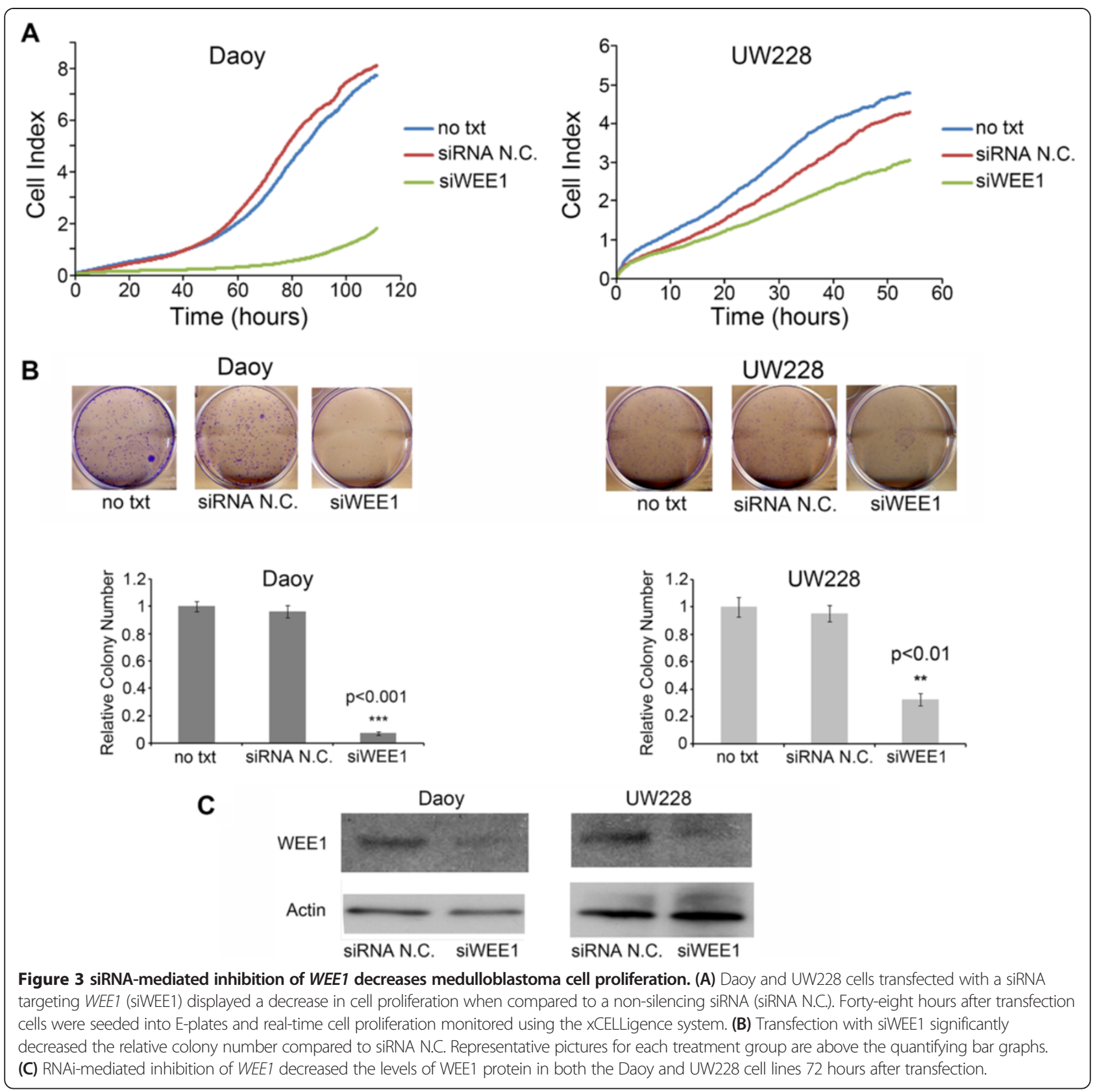

tumors were allowed to develop. Mice were treated with an oral gavage of either control solution or $30 \mathrm{mg} / \mathrm{kg}$ of MK-1775 three times a week for three weeks ( $n=10$ each group). MK-1775 was able to decrease the measurable size of the tumors compared to controls as seen in Figure 4D $(\mathrm{p}<0.005)$. A decrease in tumor volume was observed on day 63 at the termination of the study (Additional file 4: Figure S2A, p < 0.01). Recent evidence suggests that inhibition of WEE1 can lead to DNA damage. To determine if the decrease in tumor volume may have resulted from increased DNA damage snap frozen tumors were probed for $\gamma \mathrm{H} 2 \mathrm{AX}$, a marker of DNA double strand breaks. An increase in $\gamma \mathrm{H} 2 \mathrm{AX}$ protein was seen in the MK-1775 treated tumors when compared to the control tumors at day 63 (Additional file 4: Figure S2B). There was no change in the phosphorylation of CDK1, WEE1 or in the total levels of CDK1 or WEE1 in the tumor samples (data not shown) as expected. The tumors had only been treated with MK-1775 during the initial part of the study and we would not expect a transient marker like the phosphorylation of CDK1 to persist. These studies establish the proof of principle that MK-1775 is a viable candidate for medulloblastoma therapy. These data are further supported by recent studies demonstrating the single agent activity of MK-1775 in many different tumor cell lines [32]. 


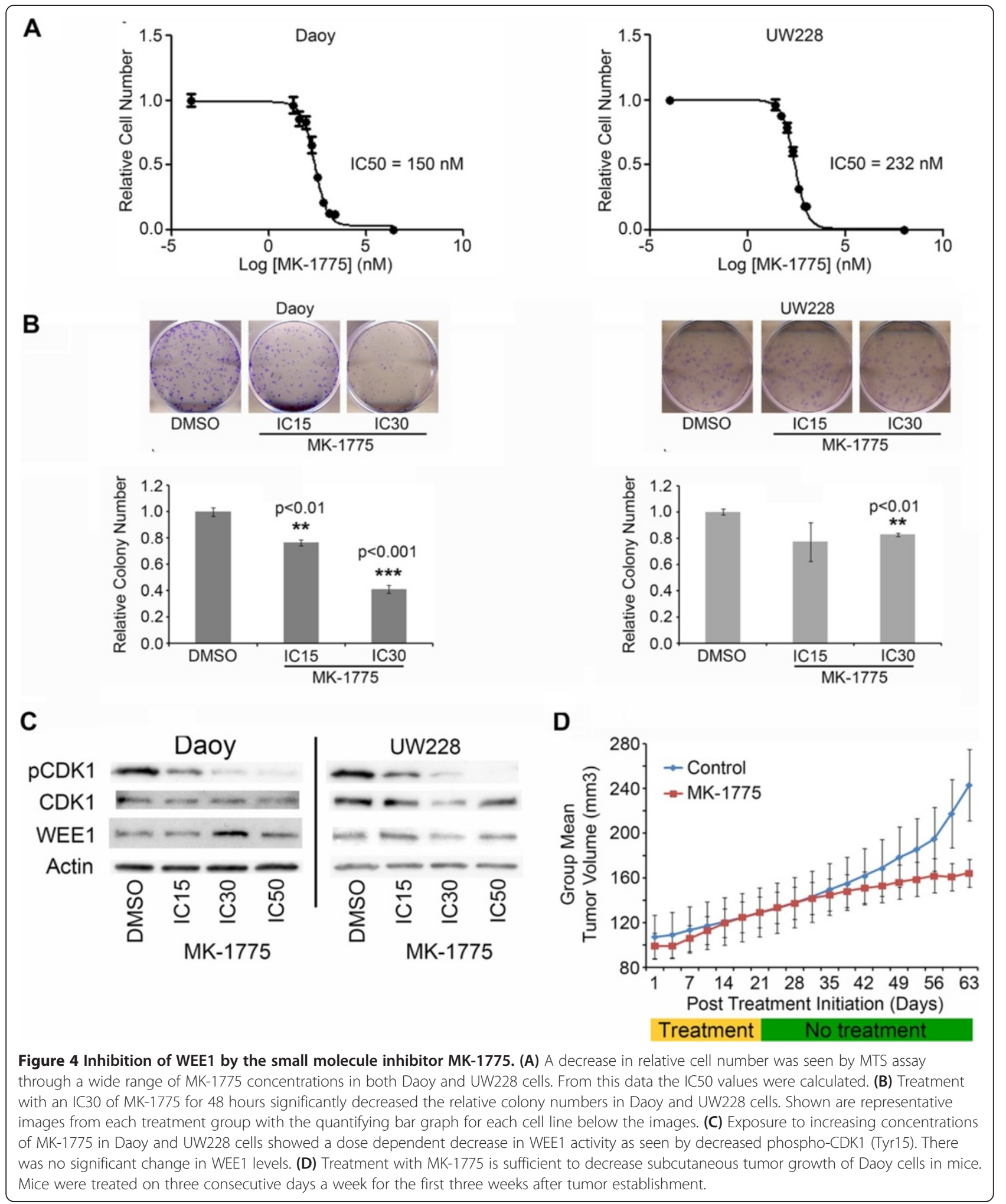

MK-1775 acts in synergy with cisplatin

Cisplatin forms the backbone of most medulloblastoma chemotherapy protocols. Among the adverse effects of cisplatin are cytotoxicity, nephrotoxicity and severe bone marrow suppression. Thus drugs that can synergize with cisplatin and facilitate a decrease in this chemotherapy are of high value. We examined the ability of MK-1775 to synergize with cisplatin. Daoy cells were exposed to 
varying concentrations of MK-1775 and cisplatin for 72 hours and the effect on cellular proliferation was evaluated by MTS assay. The results were analyzed using the Bliss Additivity model and show a synergistic relationship between cisplatin and MK-1775 at a concentration as low as $50 \mathrm{nM}$ of $\mathrm{MK}-1775$ (Figure $5 \mathrm{~A}$ ). The effect of MK-1775 on the IC50 value for cisplatin was also investigated using Daoy cells. Daoy cells have an IC50 value of $593 \mathrm{nM}$ for cisplatin alone by MTS assay. Figure $5 \mathrm{~B}$ shows a decrease in the IC50 value of cisplatin down to $470 \mathrm{nM}$ and $406 \mathrm{nM}$ when Daoy cells were treated with cisplatin and either $50 \mathrm{nM}$ or $100 \mathrm{nM}$ of MK-1775 respectively.

\section{MK-1775 induces caspase-mediated apoptosis in medulloblastoma}

To determine if the suppression of cellular growth was a result of cell death, we evaluated the impact of MK-1775 on apoptosis in medulloblastoma cells. The Daoy cell line was exposed to an IC30 of MK-1775, an IC25 of cisplatin, or both for 48 hours. The cells were subsequently stained with Guava Nexin reagent for Annexin
$\mathrm{V}$ expression and analyzed using a flow cytometer. Treatment with MK-1775 alone for 48 hours caused an increase in apoptotic cells (Figure 5C). Moreover, a significant increase in the percentage of Annexin $\mathrm{V}$ positive cells was seen when cells were treated with both MK-1775 and cisplatin compared to treatment with either drug alone $(\mathrm{p}<0.01)$. The increase in apoptotic cells can also be seen by increased caspase 3 staining in the immunofluorescence images in Additional file 5: Figure S3.

\section{MK-1775 treatment produces DNA damage and inhibits repair of cisplatin-induced DNA damage}

The DNA damage pathway plays an important part for the survival of tumor cells, especially after treatment with DNA damaging agents. To examine the impact of MK1775 on medulloblastoma cells, we analyzed for DNA damage by staining with an antibody against $\gamma \mathrm{H} 2 \mathrm{AX}$ and co-staining of the nuclei with DAPI (Figure 6A). $\gamma \mathrm{H} 2 \mathrm{AX}$ is recruited to sites of damaged DNA and is removed once DNA is repaired. After 6 hours of treatment with an IC30 of MK-1775 and/or an IC25 of cisplatin both significantly

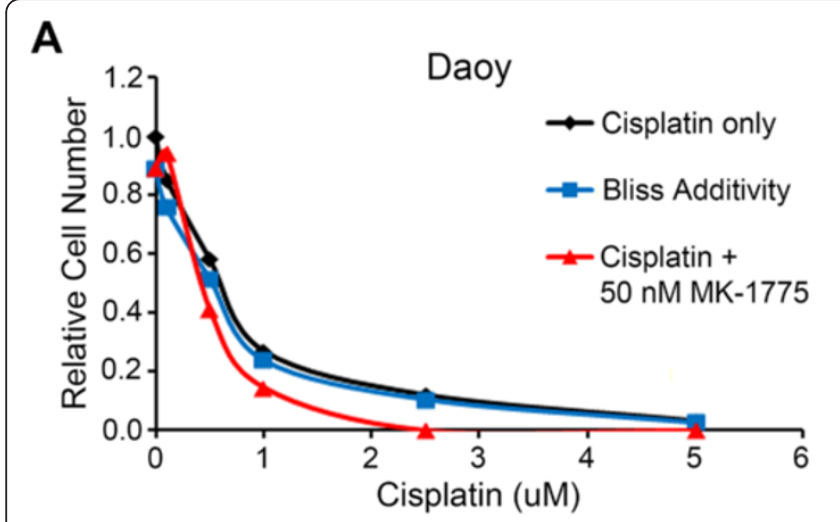

B
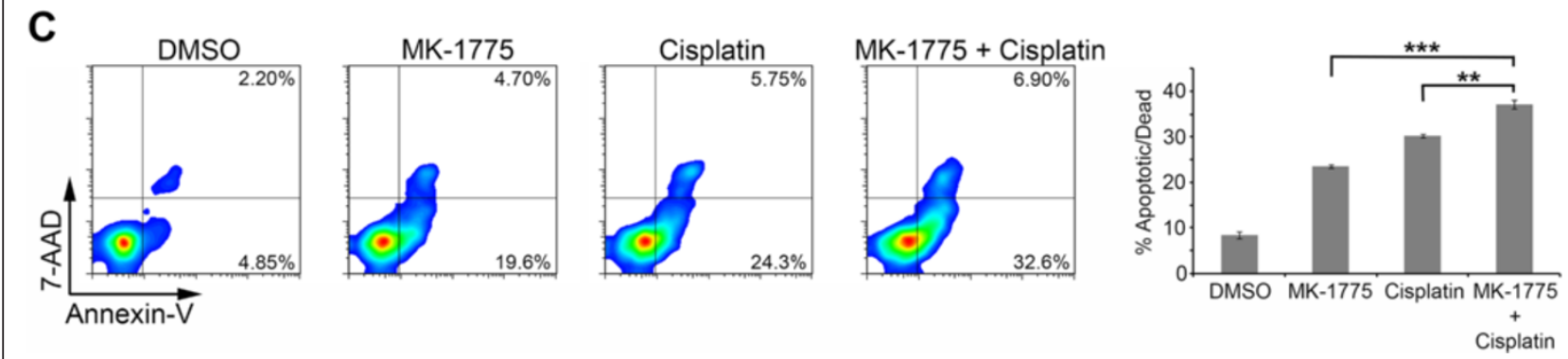

Figure 5 MK-1775 acts synergistically with cisplatin. (A) Line graph depicting the effect of different cisplatin doses with or without treatment of MK-1775 on Daoy relative cell number by MTS assay. The black line represents cells treated only with cisplatin. The blue line depicts the calculated effect of cisplatin and 50 nM MK-1775 using the Bliss Additivity model. The red line represents the observed effect of cisplatin and 50 nM MK-1775 combination treatments. (B) The bar graph depicts the calculated IC50 value by MTS assay of cisplatin in Daoy cells exposed only to cisplatin (0 nM MK-1775) or in combination with 50 nM MK-1775 or 100 nM MK-1775. (C) Representative flow cytometry plots depict the effect on apoptosis resulting from 48 hours of drug treatment in Daoy cells following staining with Guava Nexin reagent. The lower right and upper right quadrants show cells that are Annexin $\mathrm{V}$ positive and thus considered apoptotic or dead. The bar graph on the right quantifies the average percent of cells apoptotic or dead in these two quadrants for the replicate samples. The combination of an IC25 of cisplatin with an IC30 of MK-1775 increased the percentage of apoptotic cells. ${ }^{* *} p<0.01 ;{ }^{* *} p<0.001$. 


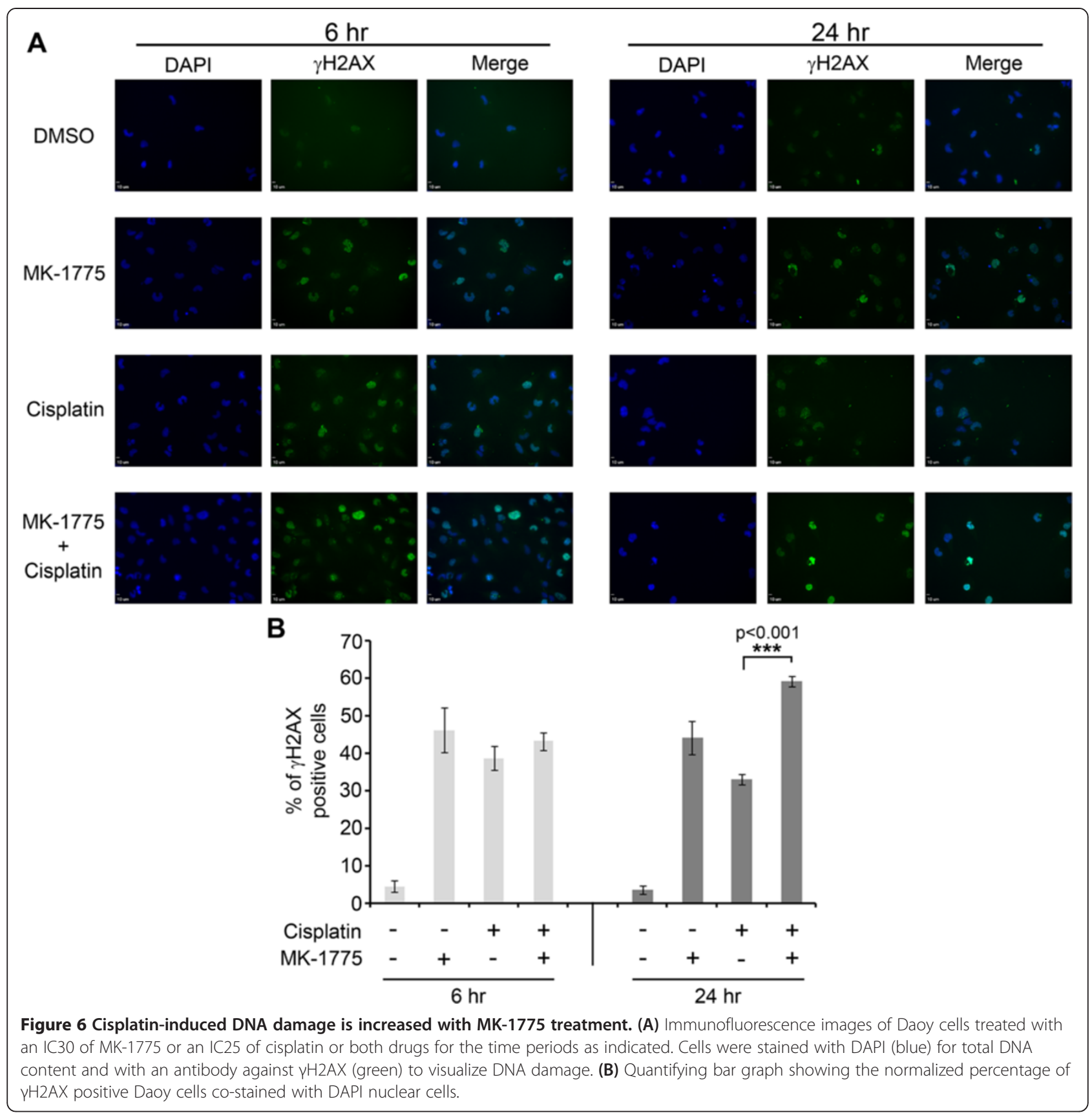

induced DNA damage as measured by $\gamma \mathrm{H} 2 \mathrm{AX}$ foci in Daoy cells (Figure 6A). However there was no significant difference between the percentages of $\gamma \mathrm{H} 2 \mathrm{AX}$ positive cells in cisplatin alone or MK-1775 treated cells for 6 hours (Figure 6B). We then measured existing DNA damage after treatment with cisplatin, MK-1775 or both for 24 hours. While there was a decrease in $\gamma \mathrm{H} 2 \mathrm{AX}$ foci in cisplatin alone treated cells compared to 6 hour treatment, there was an increase in $\gamma \mathrm{H} 2 \mathrm{AX}$ foci in cells treated with both MK-1775 and cisplatin. These data indicate that adding MK-1775 inhibits DNA damage repair seen after 24 hours of treatment with cisplatin alone.

\section{Discussion}

Medulloblastoma is a clinically and genomically heterogeneous disease. This heterogeneity creates a major challenge in developing novel therapy and improving outcomes in medulloblastoma using traditional approaches. Thus, novel approaches that more rapidly identify and validate mediators of cell survival have the potential to dramatically alter our understanding of cellular biology and develop novel therapeutic strategies. We hypothesized that escape pathways on which medulloblastoma cells are dependent for survival would represent ideal targets for therapy. To identify these pathways we integrated descriptive and 
functional genomics to objectively elucidate new targets for rationale therapy development. We identified a medulloblastoma specific kinase expression profile that can provide insight into future kinase inhibition therapeutic strategies. Next using RNAi functional genomics targeting the kinome we identified key molecules that mediate medulloblastoma cell proliferation. Integration of these data suggests that the mitotic cell cycle checkpoint is a critical determinant of medulloblastoma tumorigenesis. In particular we identified WEE1 as a potential therapeutic target. A combination of in vitro and in vivo studies clearly indicates that WEE1 mediates medulloblastoma tumorigencity and represents a novel therapeutic target. These data demonstrate the power of integrating descriptive and functional genomics to identify novel therapeutic targets. Using a similar kinomewide RNAi screen Guerreiro et al., previously identified PI3K p110y as a molecule that demonstrated synthetic lethality with cisplatin in medulloblastoma [33]. Together with our data these two studies demonstrate the utility of functional genomics to identify novel therapeutic targets in medulloblastoma.

Cell cycle checkpoint genes have previously been implicated in medulloblastoma [34-36]. However to our knowledge this is the first report in medulloblastoma to document the functional importance of mitotic kinases and in particular WEE1. WEE1 controls the G2-M transition by catalyzing the inhibitory phosphorylation of CDK1 thus preventing the Cyclin B-CDK1 complex from driving cells into mitosis [37]. The G2-M cell cycle checkpoint is critical for eukaryotic organisms ensuring cells do not initiate mitosis before DNA damage is repaired [38]. WEE1 is a key regulator of this process $[37,28]$. Thus abrogation of the G2-M checkpoint leading to premature mitotic entry and subsequent cell death by mitotic catastrophe has emerged as a promising new therapeutic strategy $[39,38]$.

WEE1 over expression is associated with several types of cancer [38]. More recently an in silico analysis of a large data set of glioblastoma identified WEE1 as over expressed and a key regulator of mitotic catastrophe [27]. Similarly a functional genomic approach in acute myeloid leukemia also identified WEE1 as a key regulator of chemotherapy sensitivity [40]. This study shows that WEE1 is over expressed and functionally important in medulloblastoma. Our data show that the small molecule inhibitor of WEE1, MK-1775, is a potent inhibitor of medulloblastoma cell growth and synergizes with cisplatin to decrease cell proliferation in vitro. Cisplatin in combination with MK-1775 treatment induces an increase in the percentage of cells in the $S$ and G2-M phases of the cell cycle (Additional file 6: Figure S4A) and subsequently leads to more DNA damage as measured by $\gamma \mathrm{H} 2 \mathrm{AX}$ foci than DNA damage induced by cisplatin alone. Similarly an increase in mitosis was demonstrated in the combination treatment by increased phospho-H3 staining as seen in Additional file 6: Figure S4B and C. Interestingly, MK-1775 as a single agent potently inhibits medulloblastoma tumor growth in vivo. These data could be explained by the fact that MK-1775 induces DNA damage and genomic instability. MK-1775 has recently been shown to inhibit growth of sarcoma cells and glioblastoma cells among many other tumor types $[31,41,42]$.

Inhibition of WEE1 has largely been investigated in the context of abnormal p53 function, given that cells with impaired p53 function are highly dependent on the G2-M checkpoint to maintain genomic integrity [43]. For example studies have demonstrated that cells with dysfunctional p53 can be sensitized to DNA damage by impairing the G2-M checkpoint through inhibition of WEE1 [30]. Our data showing synergy of WEE1 inhibition in combination with cisplatin were generated in a medulloblastoma cell line that has nonfunctional p53. A more thorough investigation with additional functional p53, nonfunctional p53 and isogenic cell lines are needed to determine the role of p53 on the effectiveness of WEE1 inhibition to sensitize cells to DNA damaging agents.

We have demonstrated that WEE1 inhibition sensitizes medulloblastoma cells to cisplatin in vitro. However it is unlikely that all patients with medulloblastoma will respond in a similar manner. Thus, it will be important to develop suitable biomarkers to predict which patients may benefit the most from such a therapeutic strategy. Detailed animal modeling of medulloblastoma with assessment of potential biomarkers as well as pharmacokinetics will provide further data in evaluating the effectiveness of WEE1 inhibition in conjunction with cisplatin in eradicating medulloblastoma cells in vivo and prolonging patient survival.

Importantly, MK-1775 is a well-tolerated drug with low dose limiting toxicities [44]. Clinical trials are now underway for MK-1775 including combination with carboplatin for ovarian cancer (NCT01164995) and combination with gemcitabine, cisplatin or carboplatin in advanced solid tumors (NCT00648648). Because MK-1775 crosses the blood brain barrier it is a promising agent for brain tumor therapy. In fact a Phase I trial in glioblastoma multiforme in adults is currently underway (NCT01849146) as well as a newly initiated trial for diffuse intrinsic pontine glioma in children (NCT01922076). For medulloblastoma therapy we envision a strategy of administering MK-1775 thrice weekly for 3 weeks with each cycle of cisplatin. Thus once the maximum tolerated dose is obtained in a Phase I trial, we would propose a Phase II/III trial of cisplatin based therapy (the current standard of care) compared to cisplatin plus MK-1775. 
In summary our data supports a role for WEE1 in regulating the G2-M checkpoint in medulloblastoma and validated WEE1 as a therapeutic target. We demonstrate that a clinically relevant small molecule inhibitor, MK-1775, is potent in the inhibition of medulloblastoma tumor growth in vivo. In light of our data and that of others, in combination with the good positive safety profile, we suggest that MK-1775 is an exciting new agent in the treatment of pediatric brain tumors, particularly medulloblastoma.

\section{Additional files}

Additional file 1: Table S1. Dysregulated cell cycle-related kinases in medulloblastoma.

Additional file 2: Table S2. Genes that attenuate medulloblastoma cell growth.

Additional file 3: Figure S1. Inhibition of cell cycle-related kinases decreased Daoy cell proliferation. Shown are five select kinases demonstrating a decrease in medulloblastoma proliferation by MTS assay. The bar graphs show the effect of the three separate siRNAs from the kinase screen on relative cell number after being normalized to the non-silencing control siRNA (siRNA N.C.). ${ }^{* *} p<0.001$.

Additional file 4: Figure S2. MK-1775 treatment decreases tumor volume and increases DNA damage in Daoy xenografts. (A) The graph depicts the average subcutaneous tumor volume in mice treated with the vehicle control DMSO or MK-1775 at the termination of the study. (B) Western blot analysis of tumors isolated at the termination of the study demonstrates an increase in $\mathrm{YH} 2 \mathrm{AX}$ protein for mice treated with MK1775 when compared to control.

Additional file 5: Figure S3. MK-1775 induces caspase 3 activation in medulloblastoma cells. Immunofluorescence images of Daoy cells treated with an IC30 of MK-1775 or an IC25 of cisplatin or both drugs are shown. Total DNA content is visualized by blue DAPI staining and caspase 3 is shown by green staining.

Additional file 6: Figure S4. Combination treatment with MK-1775 and cisplatin increases mitosis in Daoy cells. (A) Cell cycle analysis of Daoy cells treated with an IC30 of MK-1775, an IC25 of cisplatin or both for 24 hours. An increase in the percentage of cells in S and G2-M phases was observed with combination treatment. (B) Representative immunofluorescence images of Daoy cells treated with an IC30 of MK-1775, an IC25 of cisplatin, or both for 24 hours. Blue DAPI staining demonstrates nucleated cells and green staining depicts phospho-H3. (C) Quantitation of phospho-H3 positive cells in each treatment group normalized to DAPI.

\section{Competing interest}

The authors declare that they have no competing interests.

\section{Authors' contribution}

PSH: Performed siRNA screening, designed experiments and wrote the manuscript. SV: Performed immunofluorescence assays and assisted in reviewing the manuscript. IA: Performed cell proliferation assays. DKB and BC: Performed computational analysis. IB: Performed immunoblotting. AMD and MDT: Obtained and analyzed the second large cohort of medulloblastoma samples. PR: Synthesized MK-1775. NKF and RV: obtained medulloblastoma patient samples, co-designed and conceived experiments and wrote paper. All authors read and approved the final manuscript.

\section{Acknowledgements}

This work was supported by the National Institutes of Health (K08NS059790 to RV); The Hyundai Hope on Wheels Scholar Award (to RV); the Morgan Adams Foundation (to RV and NKF) and the Childhood Brain Tumor Foundation (to RV).

\section{Funding}

NINDS K08NS59790 (RV), Hyundai Hope on Wheels (RV), Morgan Adams Foundation (RV, NKF), Childhood Brain Tumor Foundation (RV).

\section{Author details}

'Department of Pediatrics and Section of Pediatric Hematology/Oncology/ BMT, Children's Hospital Colorado and University of Colorado Denver, Anschutz Medical Campus, 12800 E 19th Ave, Mail Stop 8302, Aurora, CO 80045, USA. ${ }^{2}$ Department of Neurosurgery, Children's Hospital Colorado and University of Colorado Denver, Anschutz Medical Campus, 12800 E 19th Ave, Mail Stop 8302, Aurora, CO 80045, USA. ${ }^{3}$ Department of Pharmaceutical Sciences, Skaggs School of Pharmacy and Pharmaceutical Sciences, University of Colorado Denver, Anschutz Medical Campus, 12850 E Montview Blvd, Mail Stop C238, Aurora, CO 80045, USA. ^ Division of Neurosurgery, Arthur \& Sonia Labatt Brain Tumour Research Centre, The Hospital for Sick Children, 555 University Ave, Rm 1503, Toronto, ON M5G 1X8, Canada.

Received: 8 January 2014 Accepted: 18 March 2014 Published: 24 March 2014

\section{References}

1. Dhall G: Medulloblastoma. J Child Neurol 2009, 24(11):1418-1430. doi:10.1177/0883073809341668

2. Packer RJ: Progress and challenges in childhood brain tumors J Neurooncol 2005, 75(3):239-242.

3. Ellison DW, Kocak M, Dalton J, Megahed H, Lusher ME, Ryan SL, Zhao W, Nicholson SL, Taylor RE, Bailey S, Clifford SC: Definition of disease-risk stratification groups in childhood medulloblastoma using combined clinical, pathologic, and molecular variables. J Clin Oncol, 29(11):1400-1407. doi:10.1200/JCO.2010.30.2810.

4. Ris MD, Packer R, Goldwein J, Jones-Wallace D, Boyett JM: Intellectual outcome after reduced-dose radiation therapy plus adjuvant chemotherapy for medulloblastoma: a children's cancer group study. J Clin Oncol 2001, 19(15):3470-3476.

5. Mulhern RK, Palmer SL, Merchant TE, Wallace D, Kocak M, Brouwers P, Krull K, Chintagumpala M, Stargatt R, Ashley DM, Tyc VL, Kun L, Boyett J, Gajjar A Neurocognitive consequences of risk-adapted therapy for childhood medulloblastoma. J Clin Oncol 2005, 23(24):5511-5519. doi:10.1200/ JCO.2005.00.703

6. Dhall G, Grodman H, Ji L, Sands S, Gardner S, Dunkel IJ, McCowage GB, Diez B, Allen JC, Gopalan A, Cornelius AS, Termuhlen A, Abromowitch M, Sposto R, Finlay JL: Outcome of children less than three years old at diagnosis with non-metastatic medulloblastoma treated with chemotherapy on the "Head Start" I and II protocols. Pediatr Blood Canc 2008, 50(6):1169-1175. doi:10.1002/pbc.21525.

7. Northcott PA, Korshunov A, Witt H, Hielscher T, Eberhart CG, Mack S, Bouffet E, Clifford SC, Hawkins CE, French P, Rutka JT, Pfister S, Taylor MD: Medulloblastoma comprises four distinct molecular variants. J Clin Oncol, 29(11):1408-1414. doi:10.1200/JCO.2009.27.4324.

8. Cho YJ, Tsherniak A, Tamayo P, Santagata S, Ligon A, Greulich H, Berhoukim R, Amani V, Goumnerova L, Eberhart CG, Lau CC, Olson JM, Gilbertson RJ, Gajjar A, Delattre O, Kool M, Ligon K, Meyerson M, Mesirov JP, Pomeroy SL: Integrative genomic analysis of medulloblastoma identifies a molecular subgroup that drives poor clinical outcome. J Clin Oncol, 29(11):1424-1430. doi:10.1200/JCO.2010.28.5148

9. Kool M, Koster J, Bunt J, Hasselt NE, Lakeman A, van Sluis P, Troost D, Meeteren NS, Caron HN, Cloos J, Mrsic A, Ylstra B, Grajkowska W, Hartmann W, Pietsch T, Ellison D, Clifford SC, Versteeg R: Integrated genomics identifies five medulloblastoma subtypes with distinct genetic profiles, pathway signatures and clinicopathological features. PLOS ONE 2008 3(8):e3088. doi:10.1371/journal.pone.0003088

10. Thompson MC, Fuller C, Hogg TL, Dalton J, Finkelstein D, Lau CC, Chintagumpala M, Adesina A, Ashley DM, Kellie SJ, Taylor MD, Curran T, Gajjar A, Gilbertson RJ: Genomics identifies medulloblastoma subgroups that are enriched for specific genetic alterations. J Clin Oncol 2006, 24(12):1924-1931. doi:10.1200/JCO.2005.04.4974.

11. Taylor MD, Northcott PA, Korshunov A, Remke M, Cho YJ, Clifford SC, Eberhart CG, Parsons DW, Rutkowski S, Gajjar A, Ellison DW, Lichter P, Gilbertson RJ, Pomeroy SL, Kool M, Pfister SM: Molecular subgroups of medulloblastoma: the current consensus. Acta Neuropathol. doi:10.1007/ s00401-011-0922-z 
12. Packer RJ: Risk stratification of medulloblastoma: a paradigm for future childhood brain tumor management strategies. Curr Neurol Neurosci Rep, 11(2):124-126. doi:10.1007/s11910-010-0168-5.

13. Leary SE, Olson JM: The molecular classification of medulloblastoma: driving the next generation clinical trials. Curr Opin Pediatr 2012 24(1):33-39. doi:10.1097/MOP.0b013e32834ec106.

14. Gaither A, lourgenko V: RNA interference technologies and their use in cancer research. Curr Opin Oncol 2007, 19(1):50-54. doi:10.1097/ cCo.0b013e328011a8b0

15. MacKeigan JP, Murphy LO, Blenis J: Sensitized RNAi screen of human kinases and phosphatases identifies new regulators of apoptosis and chemoresistance. Nat Cell Biol 2005, 7(6):591-600. doi:10.1038/ncb1258.

16. Barbie DA, Tamayo P, Boehm JS, Kim SY, Moody SE, Dunn IF, Schinzel AC, Sandy P, Meylan E, Scholl C, Frohling S, Chan EM, Sos ML, Michel K, Mermel C, Silver SJ, Weir BA, Reiling JH, Sheng Q, Gupta PB, Wadlow RC, Le H, Hoersch S, Wittner BS, Ramaswamy S, Livingston DM, Sabatini DM, Meyerson M, Thomas RK, Lander ES et al: Systematic RNA interference reveals that oncogenic KRAS-driven cancers require TBK1. Nature 2009, 462(7269):108-112. doi:10.1038/nature08460.

17. Luo J, Emanuele MJ, Li D, Creighton CJ, Schlabach MR, Westbrook TF, Wong KK, Elledge SJ: A genome-wide RNAi screen identifies multiple synthetic lethal interactions with the Ras oncogene. Cell 2009, 137(5):835-848. doi:10.1016/j.cell.2009.05.006.

18. Whitehurst AW, Bodemann BO, Cardenas J, Ferguson D, Girard L, Peyton M, Minna JD, Michnoff C, Hao W, Roth MG, Xie XJ, White MA: Synthetic lethal screen identification of chemosensitizer loci in cancer cells. Nature 2007, 446(7137):815-819. doi:10.1038/nature05697.

19. Cole KA, Huggins J, Laquaglia M, Hulderman CE, Russell MR, Bosse K, Diskin SJ, Attiyeh EF, Sennett R, Norris G, Laudenslager M, Wood AC, Mayes PA, Jagannathan J, Winter C, Mosse YP, Maris JM: RNAi screen of the protein kinome identifies checkpoint kinase 1 (CHK1) as a therapeutic target in neuroblastoma. Proc Natl Acad Sci U S A, 108(8):3336-3341. doi:10.1073/pnas.1012351108.

20. Ding Y, Huang D, Zhang Z, Smith J, Petillo D, Looyenga BD, Feenstra K, Mackeigan JP, Furge KA, Teh BT: Combined gene expression profiling and RNAi screening in clear cell renal cell carcinoma identify PLK1 and other therapeutic kinase targets. Canc Res, 71(15):5225-5234. doi:10.1158/00085472.CAN-11-0076.

21. Xing JZ, Zhu L, Jackson JA, Gabos S, Sun XJ, Wang XB, Xu X: Dynamic monitoring of cytotoxicity on microelectronic sensors. Chem Res Toxicol 2005, 18(2):154-161. doi:10.1021/tx049721s.

22. Birks DK, Donson AM, Patel PR, Dunham C, Muscat A, Algar EM, Ashley DM, Kleinschmidt-Demasters BK, Vibhakar R, Handler MH, Foreman NK: High expression of BMP pathway genes distinguishes a subset of atypical teratoid/rhabdoid tumors associated with shorter survival. Neuro Oncol, doi:10.1093/neuonc/nor140.

23. Subramanian A, Tamayo P, Mootha VK, Mukherjee S, Ebert BL, Gillette MA, Paulovich A, Pomeroy SL, Golub TR, Lander ES, Mesirov JP: Gene set enrichment analysis: a knowledge-based approach for interpreting genome-wide expression profiles. Proc Natl Acad Sci U S A 2005, 102(43):15545-15550. doi:10.1073/pnas.0506580102.

24. Alimova I, Venkataraman S, Harris P, Marquez VE, Northcott PA, Dubuc A, Taylor MD, Foreman NK, Vibhakar R: Targeting the enhancer of zeste homologue 2 in medulloblastoma. Int J Canc 2012. doi:10.1002/ijc.27455.

25. Harris PS, Venkataraman S, Alimova I, Birks DK, Donson AM, Knipstein J, Dubuc A, Taylor MD, Handler MH, Foreman NK, Vibhakar R: Polo-like kinase 1 (PLK1) inhibition suppresses cell growth and enhances radiation sensitivity in medulloblastoma cells. BMC Canc 2012, 12:80. doi:10.1186/1471-2407-12-80.

26. El-Sheikh A, Fan R, Birks D, Donson A, Foreman NK, Vibhakar R: Inhibition of Aurora Kinase A enhances chemosensitivity of medulloblastoma cell lines. Pediatr Blood Canc, doi:10.1002/pbc.22465.

27. Mir SE, De Witt Hamer PC, Krawczyk PM, Balaj L, Claes A, Niers JM, Van Tilborg AA, Zwinderman AH, Geerts D, Kaspers GJ, Peter Vandertop W, Cloos J, Tannous BA, Wesseling P, Aten JA, Noske DP, Van Noorden CJ, Wurdinger $\mathrm{T}$ : In silico analysis of kinase expression identifies WEE1 as a gatekeeper against mitotic catastrophe in glioblastoma. Canc cell, 18(3):244-257. doi:10.1016/j.ccr.2010.08.011.

28. De Witt Hamer PC, Mir SE, Noske D, Van Noorden CJ, Wurdinger T: WEE1 kinase targeting combined with DNA-damaging cancer therapy catalyzes mitotic catastrophe. Clin Canc Res 2011, 17(13):4200-4207. doi:10.1158/ 1078-0432.CCR-10-2537.

29. Mir SE, De Witt Hamer PC, Krawczyk PM, Balaj L, Claes A, Niers JM, Van Tilborg AA, Zwinderman AH, Geerts D, Kaspers GJ, Peter Vandertop W, Cloos J, Tannous BA, Wesseling P, Aten JA, Noske DP, Van Noorden CJ, Wurdinger T: In silico analysis of kinase expression identifies WEE1 as a gatekeeper against mitotic catastrophe in glioblastoma. Canc Cell 2010, 18(3):244-257. doi:10.1016/j.ccr.2010.08.011.

30. Hirai H, Iwasawa Y, Okada M, Arai T, Nishibata T, Kobayashi M, Kimura T, Kaneko N, Ohtani J, Yamanaka K, Itadani H, Takahashi-Suzuki I, Fukasawa K, Oki H, Nambu T, Jiang J, Sakai T, Arakawa H, Sakamoto T, Sagara T, Yoshizumi T, Mizuarai S, Kotani H: Small-molecule inhibition of Wee1 kinase by MK-1775 selectively sensitizes p53-deficient tumor cells to DNA-damaging agents. Mol Canc Ther 2009, 8(11):2992-3000. doi:10.1158/1535-7163.MCT-09-0463.

31. Sarcar B, Kahali S, Prabhu AH, Shumway SD, Xu Y, Demuth T, Chinnaiyan P. Targeting radiation-induced $\mathrm{G}(2)$ checkpoint activation with the Wee-1 inhibitor MK-1775 in glioblastoma cell lines. Mol Canc Ther 2011, 10(12):2405-2414. doi:10.1158/1535-7163.MCT-11-0469.

32. Guertin AD, Li J, Liu Y, Hurd MS, Schuller AG, Long B, Hirsch HA, Feldman I, Benita Y, Toniatti C, Zawel L, Fawell SE, Gilliland DG, Shumway SD: Preclinical evaluation of the WEE1 inhibitor MK-1775 as single-agent anticancer therapy. Mol Canc Ther 2013, 12(8):1442-1452. doi:10.1158/15357163.MCT-13-0025.

33. Guerreiro AS, Fattet S, Kulesza DW, Atamer A, Elsing AN, Shalaby T, Jackson SP, Schoenwaelder SM, Grotzer MA, Delattre O, Arcaro A: A sensitized RNA interference screen identifies a novel role for the PI3K p110gamma isoform in medulloblastoma cell proliferation and chemoresistance. Mol Canc Res, 9(7):925-935. doi:10.1158/1541-7786.MCR-10-0200.

34. Uziel T, Zindy F, Sherr CJ, Roussel MF: The CDK inhibitor p18Ink4c is a tumor suppressor in medulloblastoma. Cell Cycle 2006, 5(4):363-365.

35. Whiteway SL, Harris PS, Venkataraman S, Alimova I, Birks DK, Donson AM, Foreman NK, Vibhakar R: Inhibition of cyclin-dependent kinase 6 suppresses cell proliferation and enhances radiation sensitivity in medulloblastoma cells. J Neurooncol 2012. doi:10.1007/s11060-012-1000-7.

36. Mendrzyk F, Radlwimmer B, Joos S, Kokocinski F, Benner A, Stange DE, Neben K, Fiegler H, Carter NP, Reifenberger G, Korshunov A, Lichter P: Genomic and protein expression profiling identifies CDK6 as novel independent prognostic marker in medulloblastoma. J Clin Oncol 2005, 23(34):8853-8862. doi:10.1200/JCO.2005.02.8589.

37. Russell $P$, Nurse $P$ : Negative regulation of mitosis by wee1+, a gene encoding a protein kinase homolog. Cell 1987, 49(4):559-567. doi:0092 8674(87)90458-2.

38. Janssen A, Medema RH: Mitosis as an anti-cancer target. Oncogene, 30(25):2799-2809. doi:10.1038/onc.2011.30

39. Kawabe T: G2 checkpoint abrogators as anticancer drugs. Mol Canc Ther 2004, 3(4):513-519.

40. Porter CC, Kim J, Fosmire S, Gearheart CM, van Linden A, Baturin D, Zaberezhnyy V, Patel PR, Gao D, Tan AC, Degregori J: Integrated genomic analyses identify WEE1 as a critical mediator of cell fate and a novel therapeutic target in acute myeloid leukemia. Leukemia 2012. doi:10.1038/leu.2011.392

41. Kreahling JM, Gemmer JY, Reed D, Letson D, Bui M, Altiok S: MK1775, a selective Wee1 inhibitor, shows single-agent antitumor activity against sarcoma cells. Mol Canc Ther 2012, 11(1):174-182. doi:10.1158/1535-7163. MCT-11-0529.

42. Stathis A, Oza A: Targeting Wee1-like protein kinase to treat cancer. Drug News Perspect, 23(7):425-429. doi:10.1358/dnp.2010.23.7.1490760.

43. de Carcer G, Perez de Castro I, Malumbres M: Targeting cell cycle kinases for cancer therapy. Curr Med Chem 2007, 14(9):969-985.

44. Schellens SL JH, Shapiro Gl, Pavlick AC, Tibes R, O'Day SJ, Demuth T, Viscusi J, Xu Y, Oza AM: A phase I and pharmacological study of MK-1775, a Wee1 tyrosine kinase inhibitor, in both monotherapy and in combination with gemcitabine, cisplatin, or carboplatin in patients with advanced solid tumors. J Clin Oncol 2009, 27(15S):3510.

\section{doi:10.1186/1476-4598-13-72}

Cite this article as: Harris et al:: Integrated genomic analysis identifies the mitotic checkpoint kinase WEE1 as a novel therapeutic target in medulloblastoma. Molecular Cancer 2014 13:72. 